\title{
Case Report: Missing the Bull's Eye: A Case Report of Tibialis Anterior Hernia
}

\author{
Muhammed Minhaj ${ }^{*}$ (iD, Binoy Mathew² (1)
}

1. Department of Sports Physiotherapy, Jamia Millia Islamia University, New Delhi, India.

2. Department of Physiotherapy, Composite Regional Centre for Skill Development Rehabilitation and Empowerment of Persons with Disabilities, Kozhikode, India.

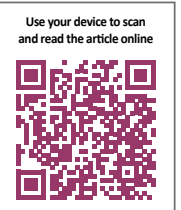

Citation Minhaj M, Mathew B. Missing the Bull's Eye: A Case Report of Tibialis Anterior Hernia. Iranian Rehabilitation Journal. 2021; 19(4):465-472. http://dx.doi.org/10.32598/irj.19.4.1458.1

doijhttp://dx.doi.org/10.32598/irj.19.4.1458.1

Article info:

Received: 14 May 2021

Accepted: 20 Sep 2021

Available Online: 01 Dec 2021

\section{Keywords:}

Athletic injuries, Hernia, Muscles, Diagnostic errors, Lower extremity, Ultrasonography

\section{ABSTRACT}

Objectives: Shin pain is a broad term used to describe lower leg pain. It is commonly seen in the athletic/sports population. Various conditions have been claimed to produce leg pain, and muscle herniation is one of these conditions. Muscle herniation is the protrusion of muscle through a defect in the overlying fascia. Although such herniation is common, it is often an underdiagnosed condition in the lower extremity. It usually occurs in athletes, especially in the anterior compartment of the leg, as a result of trauma or due to muscle hypertrophy secondary to strenuous exercise. Herniation diagnosis depends on its clinical presentation as it appears as a palpable soft tissue bulge through a fascia defect; radiological findings are used to confirm the diagnosis. Conservative treatment should be the initial approach, and surgery can be performed if conservative treatment fails.

Methods: We present a case of symptomatic anterior lower leg pain, initially diagnosed as a psychosomatic disorder by two orthopedic physicians. However, tibialis anterior hernia in this patient was confirmed after physical examination by a physiotherapist and subsequent referral for ultrasonographic evaluation to a radiologist. The patient underwent an 8-week course of conservative treatment under the supervision of a physiotherapist. This treatment significantly improved the patient's pain and function.

Results: After eight weeks of conservative treatment using Minhaj protocol for Tibialis Anterior hernia, the patient has reported improvement in pain (reduction of score from eight to two on the NPRS scale) and function. The patient returned to his previous level of physical activity following eight weeks of rehabilitation.

Discussion: Tibialis anterior hernia should be considered a differential diagnosis in chronic leg pain with palpable soft tissue protrusion. Conservative treatment can be chosen as the primary approach.

\section{* Corresponding Author:}




\section{Highlights}

- Muscle herniations are one of the causes of lower leg pain among athletes.

- Tibialis anterior hernia is the protrusion of muscle through the defect in the fascia.

- Conservative treatment should be considered as an initial treatment.

- Minhaj protocol is a new physiotherapy management protocol for tibialis anterior hernia.

\section{Plain Language Summary}

Muscle hernias are the bulging out of the muscles through the overlying membrane covering. Tibialis Anterior is a muscle seen in the lower leg. Hernia of the Tibialis hernia muscle is one of the causes of lower leg pain and is often an undetected condition in the lower leg. Due to injury and increased muscle bulk following strenuous muscle activity, it commonly occurs in athletes. Diagnosis of Tibialis Anterior hernia is mainly based on its presentation as a soft tissue bulge that can be felt under the overlying skin. The node may be enlarged by tightening the muscle and disappear by muscle relaxation. Special medical imaging studies such as Magnetic Resonance Imaging (MRI), Ultrasonography (US), and Computer Tomography are used to confirm the diagnosis of Tibialis anterior hernia. Treatment of Tibialis Anterior hernia is mainly physiotherapy consisting of rest, application of compression bandage, modification of activities, and specific exercise in a phased manner. Such treatment should be considered an initial treatment approach for Tibialis Anterior hernia, and surgery can be performed in case of failure of this approach. They presented a case history of an athlete presented to the Physiotherapy department with exercise-related lower leg pain. Detailed examination by the physiotherapist and after ultrasound scanning by a radiologist confirmed it as a Tibialis Anterior hernia. The patient has undergone eight weeks of Physiotherapy treatment and returned to the previous level of activity after eight weeks of rehabilitation.

\section{Introduction}

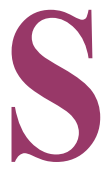

hin pain is an umbrella term often used by sports physicians, physiotherapists, and trainers to describe exercise-induced pain in the lower leg. The common features of exercise-induced lower leg pain include pain that aggravates with physical activity and relieves with rest. Other symptoms are muscle weakness, muscle cramps, muscle herniation, muscle tightness, paraesthesia, and numbness. The conditions leading to exercise-induced lower leg pain can be categorized into medial tibial stress syndrome, stress fracture, chronic exertional compartmental syndrome, nerve entrapments, artery entrapment, and muscle herniation [1]. Diagnosis of these conditions is challenging because of the absence of symptoms at rest and reappearance only during strenuous exercise. Comprehensive knowledge of lower leg conditions, mechanism of injury, relevant history collection, patient examination, and specific diagnostic tests are necessary for diagnosing these lower leg conditions.

Muscle herniation is one of the causes of lower leg pain and is not rare among athletes. It is commonly seen in the anterior compartment of the lower leg. Lower extremity herniations are also seen in the posterior compartment of the leg, lateral compartment of the leg, and anterior compartment of the thigh. It usually occurs due to a defect in the deep fascial layer of the muscle. It is mainly seen in athletes due to trauma or muscle hypertrophy secondary to strenuous muscle activity [2]. Radiological imaging technique such as Magnetic Resonance Imaging (MRI), Ultrasonography (US), and Computer Tomography (CT) plays an essential role in identifying these defects in the absence of symptoms [2]. This study aimed to provide information regarding the features of the tibialis anterior hernia and highlight conservative treatment's effectiveness in managing the condition.

\section{Case Report}

A 23-year-old man and recreational football player with 7 years' experience in playing was presented to a physiotherapist with a complaint of the exercise-induced chronic right lower leg and foot pain since at least a year ago. The patient was an undergraduate student and used to play in the college football team as a left-wing midfielder around 5 to 6 days a week. Apart from football, he used to spend at least 40 minutes in the gym. The pain became severe in the last three months, and he consulted 
two orthopedic physicians, who diagnosed his problem as a psychosomatic disorder. Clinical history showed no previous traumatic event or surgery in the lower leg. He stopped playing three months ago because of severe exercise-induced pain in his lower leg. He was also complaining of a structural bulging in the right lower leg accompanied by pain. The pain was insidious in onset, and he could barely continue to play beyond 15 minutes because of the pain. At least 30 minutes of rest was needed to relieve his pain. The patient marked $9 / 10$ pain on the NPRS scale during playing, and otherwise, it was $0 / 10$ at rest. Besides, he felt the weakness of the ankle and foot during strenuous exercise and numbness over the plantar surface of the foot after prolonged standing.

Local examination of the area at rest failed to reproduce the pain, swelling, or numbness. But a palpable gap could be felt in the anterolateral mid-portion of the right lower leg, approximately $15 \mathrm{~cm}$ below the knee joint and $3 \mathrm{~cm}$ lateral to the anterior crest of the tibia (Figure 1). No tenderness was found in the defective area. There was no variation in skin temperature or discoloration in the area. The patient confirmed that the bulging occurs in the same area of the defect. Resisted dorsiflexion reproduces the bulging through this gap. Muscle length assessment revealed moderate hamstring and calf muscle tightness. Manual Muscle Testing (MMT) showed the weakness of quadriceps and dorsiflexor muscles (MMT 4) compared to the normal leg. A fascia defect and accompanying muscle herniation were suspected after the physical examination by the physiotherapist. For confirmation, the patient was referred to a radiologist for evaluation.

The radiologist performed US imaging both in supine lying and standing position. The lying position failed to reproduce the muscle bulging, whereas bulging through the defect occurred in the standing position. US imaging detected an approximately $4 \mathrm{~cm}$ long vertical defect in the fascia and herniation of the tibialis anterior through the defect. Thus, the radiologist confirmed the diagnosis of the tibialis anterior hernia (Figure 2).

During the subsequent visit, the physiotherapist designed and prescribed an exercise protocol, Minhaj protocol, for tibialis anterior hernia (Table 1). The goal was to limit his exercise-induced pain. For reducing the load on the tibialis anterior muscle, the patient was asked to focus on mid-foot landing instead of heel strike and increase cadence by $5 \%-10 \%$ while running $[3,4]$.

The post-intervention assessment was performed two months after the initial consultation. The patient reported a beneficial effect after 8 weeks of physiotherapy treatment for his exercise-induced pain. He could play for at least 30 minutes without any lower leg symptoms. He reported a 2/10 pain on the NPRS scale (Figure 3) and mild bulging after 30 minutes of activity, and the pain got relieved with 10 minutes of rest. MMT showed grade 5 strength in quadriceps and dorsiflexor muscles. The hamstring and calf muscle length were returned to normal.

\section{Discussion}

Although muscle herniation in the lower extremity is a common condition in athletes, they are often underdiagnosed or misdiagnosed as muscle hematomas and varicosities [5]. These are the protrusions of the muscle tissue through the defect in the fascia overlying the muscle [6]. Though the incidence of tibialis anterior hernia is unknown, it is commonly reported in athletes. Muscle herniations are commonly seen in the anterolateral side of the lower leg than other sites due to superficiality, weakness, and tightness of the fascial compartment in this area [6] and its more vulnerability to traumatic injury [7]. Additionally, literature has reported the involve-

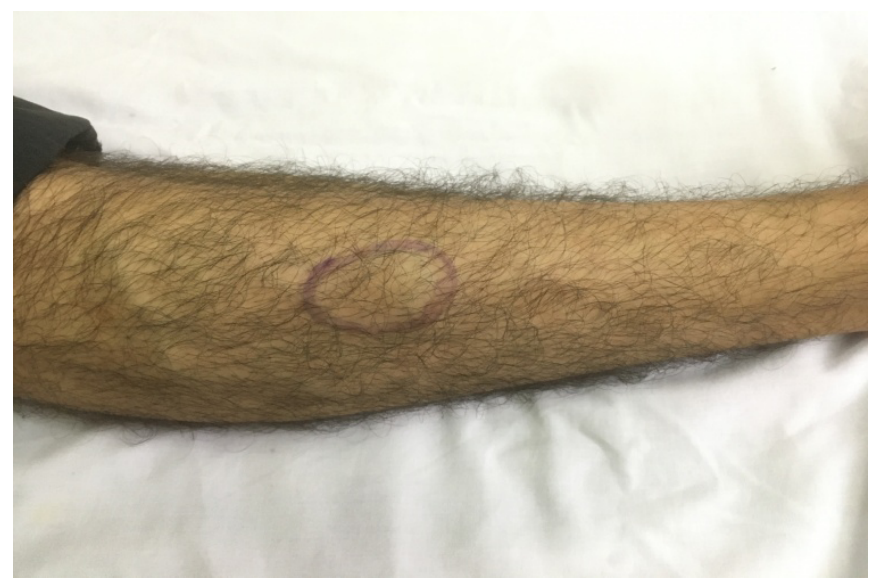

Figure 1. Location of the fascial defect

Iranian Rehabilitation Journal 

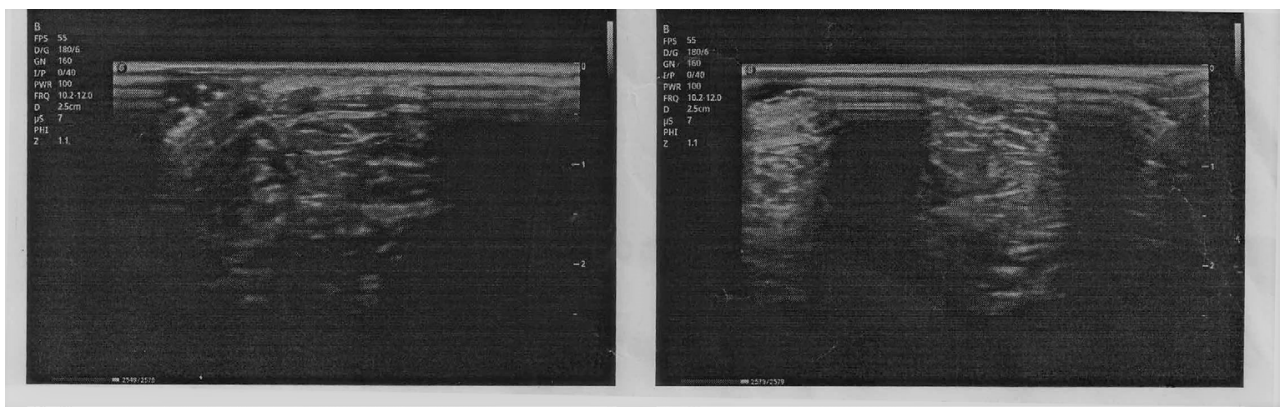

Пranian Rehabilitation \ournal

Figure 2. Ultrasonography image revealing the loss of continuity of the fascia and protrusion of the tibialis anterior muscle

ment of herniation in the peroneal compartment, tibialis posterior compartment, and quadriceps compartment [2, 7]. According to many studies, muscle herniations are divided into two categories [7]: constitutional and traumatic. Constitutional herniation occurs due to generalized weakness in the muscle fascia. Traumatic herniation occurs secondary to other injuries such as closed fracture along with a fascial tear, penetrating trauma, and indirect injury caused by excessive force applied on an already contracted muscle [7].

Diagnosis of tibialis anterior hernia is based on its clinical presentation as a soft tissue bulge that protrudes through the area of fascial defect. Bulge may enlarge with the contraction of the affected muscle and disappear when the muscle is relaxed [8]. Most of the time, the patients present with an asymptomatic soft tissue protrusion, but they may complain of associated pain, cramps, and tenderness with strenuous exercise [8]. Sensory symptoms may also arise in case of nerve involvement. Hernia commonly occurs in adolescent and adult populations who regularly participate in higher muscular activity and may be seen unilaterally or bilaterally. Resisted dorsiflexion can be used to reproduce the protru- sion of muscle fibers. An underlying fascial defect can be detected by palpation. Thus, a detailed physical examination is needed to diagnose these conditions. Radiological imaging techniques such as MRI, US, and CT play a crucial role in diagnosis confirmation [2].

Dynamic ultrasound is widely used to evaluate muscle hernia as it can detect the bulging while performing the muscle contraction and reduction of herniation during muscle relaxation. Because of the difficulty in feeling the bulge, the skin over the defective area will be marked before the ultrasonography procedure. Evaluation can be performed in standing and lying positions, and additional contraction of the suspected muscles reproduces the bulging through the fascial defect. Low pressure while moving US transducer is preferred as the high pressure may alter the presentation of hernia, and focus should be given in and around the field of skin marking [9]. Examination by the MRI is not recommended in detecting the muscle hernia due to its cost and time consuming and the dynamic nature of muscle hernia. Treatment of the tibialis anterior hernia is mostly conservative, including rest, activity modification, compression bandage, and exercise $[2,4,10]$. A paper published in 2020 by A.

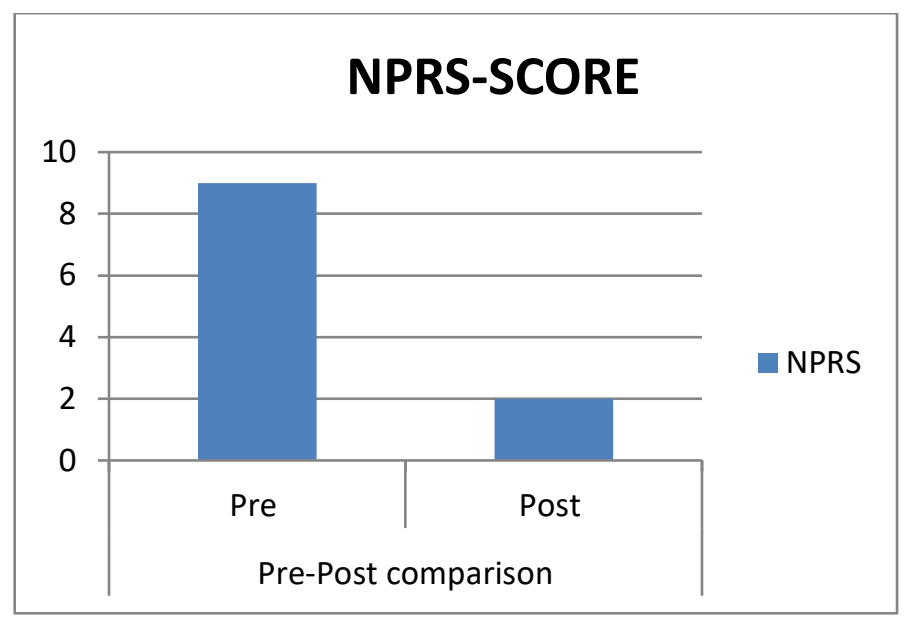

Figure 3. Pre-post pain comparison

Iranian Rehabilitation Journal 
Table 1. Minhaj protocol for tibialis anterior hernia

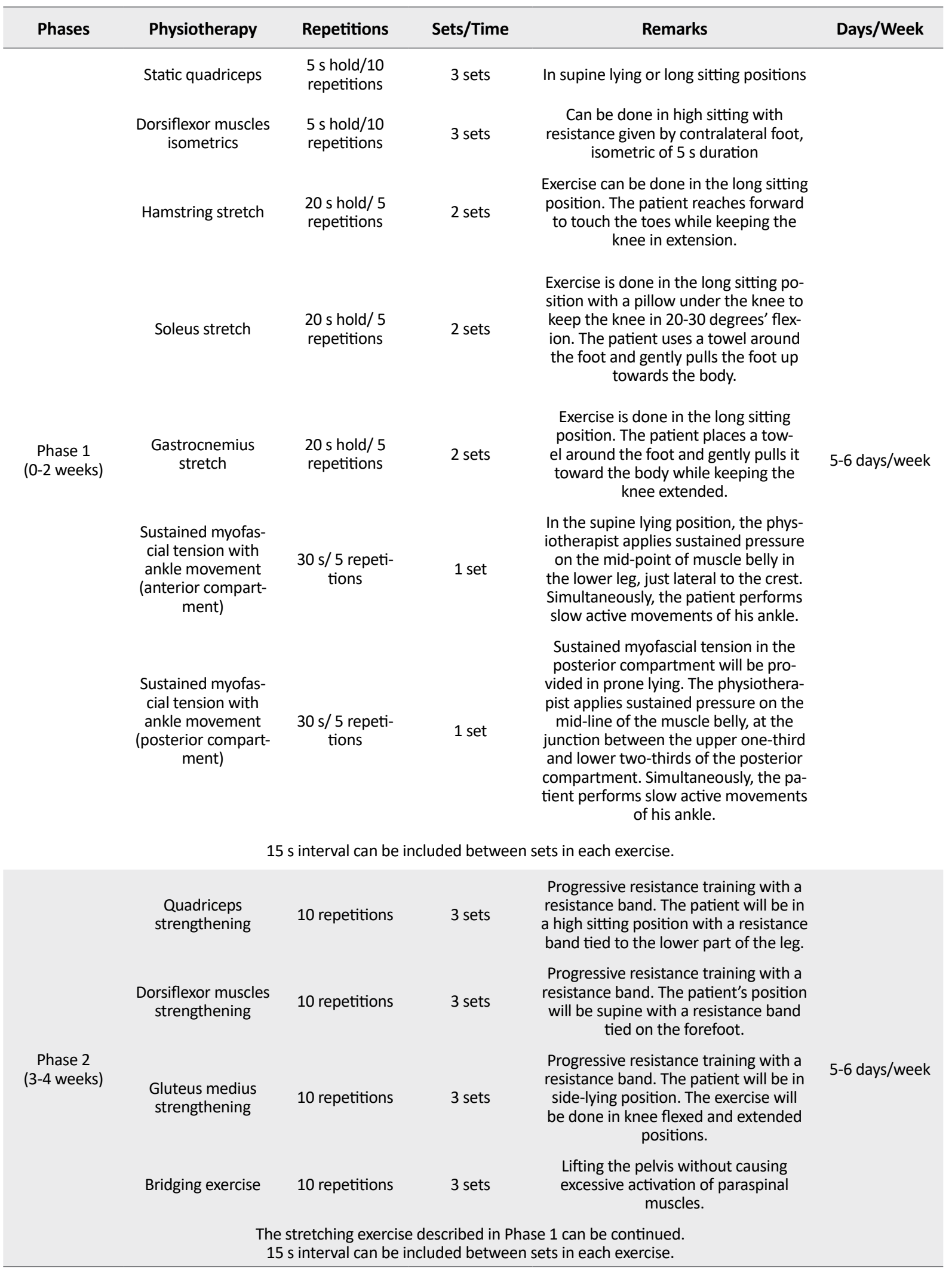




\begin{tabular}{|c|c|c|c|c|c|}
\hline Phases & Physiotherapy & Repetitions & Sets/Time & Remarks & Days/Week \\
\hline \multirow{5}{*}{$\begin{array}{l}\text { Phase } 3 \\
\text { (5-6 weeks) }\end{array}$} & $\begin{array}{l}\text { Hamstring ec- } \\
\quad \text { centrics } \\
\text { (Nordic exercise) }\end{array}$ & 7 repetitions & 3 sets & $\begin{array}{l}\text { The patient will be in a kneeling posi- } \\
\text { tion. Keeping his head, trunk, and hip } \\
\text { upright, the patient moves forward by } \\
\text { extending at the knee while the phys- } \\
\text { iotherapist stabilizes his lower legs. }\end{array}$ & \multirow{4}{*}{$\begin{array}{c}2-3 \text { days/week } \\
\text { with an interval } \\
\text { between ses- } \\
\text { sions }\end{array}$} \\
\hline & $\begin{array}{l}\text { Quadriceps ec- } \\
\text { centrics } \\
\text { (Reverse Nordic } \\
\text { exercise) }\end{array}$ & 7 repetitions & 3 sets & $\begin{array}{l}\text { The patient will be in a kneeling posi- } \\
\text { tion. Keeping head, trunk, and hip } \\
\text { upright, the patient moves backward, } \\
\text { bending at the knee. }\end{array}$ & \\
\hline & $\begin{array}{l}\text { Dorsiflexor muscles } \\
\quad \text { eccentrics }\end{array}$ & 7 repetitions & 3 sets & $\begin{array}{l}\text { Supine lying position with the weight } \\
\text { applied on foot. The patient does plan- } \\
\text { tar flexion with eccentric activation of } \\
\text { dorsiflexor muscles. }\end{array}$ & \\
\hline & \multirow[t]{2}{*}{$\begin{array}{l}\text { Calf muscle ec- } \\
\text { centrics }\end{array}$} & 7 repetitions & 3 sets & $\begin{array}{l}\text { Performed while standing on a foot- } \\
\text { step with only forefoot contacting the } \\
\text { surface. Allow controlled dorsiflexion } \\
\text { with eccentric activation of plantar } \\
\text { flexors. }\end{array}$ & \\
\hline & & \multicolumn{3}{|c|}{$\begin{array}{l}\text { Phase } 2 \text { exercises and stretching are to be continued. } \\
\text { inute interval can be included between sets in each exercise. }\end{array}$} & \\
\hline \multirow{5}{*}{$\begin{array}{l}\text { Phase } 4 \\
\text { (7-8 weeks) }\end{array}$} & Power skip & 10 repetitions & 2 sets & $\begin{array}{l}\text { It is performed with a } 15 \mathrm{~m} \text { forward } \\
\text { movement. Skip as high as possible. }\end{array}$ & \multirow{5}{*}{$\begin{array}{l}\text { 2-3 days/week } \\
\text { with interval } \\
\text { between ses- } \\
\text { sion }\end{array}$} \\
\hline & Single-leg hop & 10 repetitions & 2 sets & $\begin{array}{l}\text { Single-leg stand with slight knee flex- } \\
\text { ion. Hop as long as possible. }\end{array}$ & \\
\hline & Double-leg hop & 10 repetitions & 2 sets & $\begin{array}{l}\text { Double-leg stand with slight knee } \\
\text { flexion. Hop as long as possible. }\end{array}$ & \\
\hline & Squat Jump & 10 repetitions & 2 sets & $\begin{array}{l}\text { Double-leg stand with slight knee } \\
\text { flexion. Jump as high as possible. }\end{array}$ & \\
\hline & \multicolumn{4}{|c|}{ One minute interval can be included between sets in each exercise. } & \\
\hline
\end{tabular}

Masoumi and G. Ramogida [4] suggested a specific exercise pattern combining progressive isometric, concentric, eccentric, and sports-specific exercises to improve symptoms related to muscle hernia in the sports population. According to this paper, giving supine isometrics to the tibialis anterior for the first two weeks will help reduce pain because of the analgesic effect of isometric exercise and also promote motor neuron recruitment. They recommend concentric exercise in supine and weightbearing positions for the next two weeks, followed by eccentric strengthening to produce maximum strength in elongated position and to adapt with the elastic force.

The last stage in this exercise pattern consists of plyometric drills specific to sports performance. It was reported that the patient would be completely recovered and allowed to participate in sports after eight weeks of rehabilitation. We incorporated similar components in the current protocol with some modifications. To improve the strength of the quadriceps and dorsiflexor muscles, we advised the patient to do progressive resistance training for these muscles. Moreover, the literature supports the use of concentric exercise to increase the strength of agonists and improve the recruitment of motor neurons. We included stretching of the hamstring and calf to improve the flexibility of muscles. Stretching was found to have a beneficial effect on muscle flexibility [11]

Masood et al. [11] reported that at least $15 \mathrm{~s}$ stretch is needed to benefit muscle extensibility. Additionally, eccentric strengthening was also given along with these exercises. Studies have described the effect of eccentric training on muscle architecture and strength [12]. Eccentric training leads to an increase in fascicle length, improvement in muscle thickness, and increase in muscle strength. Plyometrics was also performed in the final 
stage of our protocol. The protocol was beneficial as the patient reported improvement in exercise-induced pain and function in his lower leg. He could return to his previous level of sports activity after 8 weeks of conservative treatment.

If the conservative treatment fails to reduce the symptom, surgical procedures can manage the hernia. The traditional method of direct fascial defect closure has lost its popularity in modern medicine due to the recurrence of muscle hernia [13]. Recent research suggests various surgical methods to repair muscle herniation, even though the evidence-based guidelines for surgery are lacking. The use of local fascial flap [14], graft jacket [15], and synthetic patches [2] are some of the surgical methods mentioned in published papers for the repair of fascial defects. The result of this case study could not be generalized as it was performed in a single subject.

\section{Conclusion}

Tibialis anterior hernia should be considered a differential diagnosis in chronic leg pain associated with palpable soft tissue protrusion. A detailed physical examination and confirmation through MRI, CT, or US are needed to diagnose tibialis anterior hernia. Conservative treatment should be primarily considered, and surgery may be a choice only if conservative measures fail.

\section{Ethical Considerations}

\section{Compliance with ethical guidelines}

Informed consent was obtained from the patient. This case study was conducted following the ethical standards of the Helsinki declaration.

\section{Funding}

This research received no specific grant from any funding agency in the public, commercial, or not-for-profit sectors.

\section{Authors' contributions}

All authors equally contributed to preparing the article.

\section{Conflict of interest}

The authors declared no conflict of interest.

\section{Acknowledgments}

We thank Mr. Sajesh (Sports Physiotherapist) for his opinion about the condition and Ms. Dimple Khurana
(Sports Physiotherapist) for spending her valuable time on language editing.

\section{References}

[1] Barton CI, Bonanno DR, Carr J, Neal BS, Malliaras P, Franklyn-Miller A, Menz HB. Running retraining to treat lower limb injuries: A mixed-methods study of current evidence synthesised with expert opinion. British Journal of Sports Medicine 2016; 50(9):513-26. [DOI:10.1136/ bjsports-2015-095278]

[2] Ceyhan AM, Chen W, Yener M, Yildirim M, Yesildag A, Akkaya VB. Bilateral tibialis anterior muscle herniation simulating a soft tissue tumour in a young amateur football player Australasian Journal of Dermatology. 2010; 51(2):142-4 [DOI:10.1111/j.1440-0960.2009.00615.x]

[3] Dönmez G, Evrenos MK, Cereb M, Karanfil Y, Doral MN. Double layer repair of tibialis anterior muscle hernia in a soccer player: A case report and review of the literature. Muscles, Ligaments and Tendons Journal. 2015; 5(4):331-4. [DOI:10.11138/mltj/2015.5.4.331]

[4] Dyson K, Palan J, Mangwani J. Bilateral non-traumatic lower leg fascial defects causing peroneal muscle herniation and novel use of a GraftJacket to repair the fascial defect. Journal of Clinical Orthopaedics and Trauma. 2019; 10(5):879-83. [DOI:10.1016/j.jcot.2018.09.009]

[5] Edwards PH, Wright ML, Hartman JF. A Practical Approach for the Differential Diagnosis of Chronic Leg Pain in the Athlete. 1241-9. [DOI:10.1177/0363546505278305]

[6] Gérard R, Gojon L, Decleve P, Van Cant J. The effects of eccentric training on biceps femoris architecture and strength A systematic review with meta-analysis. Journal of Athletic Training. 2020; 55(5):501-4. [DOI:10.4085/1062-6050-194-19]

[7] Hullur H, Salem Y, Al-Khalifa J, Salem A. Tibialis anterior muscle hernia: Rare but not uncommon. BMJ Case Reports. 2016; 2016:2-4. [DOI:10.1136/ bcr-2016-217569]

[8] Khaladkar SM., Kondapavuluri SK, Kamal A, Kalra R, Kamal V. Detection of myofascial herniation on dynamic sonog raphy and magnetic resonance imaging. Case Reports in Radiology. 2016; 2016(c):1-5. [DOI:10.1155/2016/4245189]

[9] Marchalik D, Lipsky A, Petrov D, Harvell JD, Milgraum SS, Dermatologic presentations of orthopedic pathologies. American Journal of Clinical Dermatology. 2012; 13(5):293-310. [DOI:10.2165/11595880-000000000-00000]

[10] Masood K, Riaz H, Ghous M, Iqbal M. Comparison between dynamic oscillatory stretch technique and static stretching in reduced hamstring flexibility in healthy population: A single blind randomized control trial. Journal of the Pakistan Medical Association. 2020; 70(11):1908-12. [DOI:10.5455/ JPMA.24472]

[11] Masoumi A, Ramogida G. Tibialis anterior herniation-a rare clinical entity: A case report and review of the literature. Journal of the Canadian Chiropractic Association. 2020; 64(1):88-91. [PMID] [PMCID] 
[12] Miniaci A, Rorabeck $\mathrm{CH}$. Tibialis anterior muscle hernia: A rationale for treatment. Canadian Journal of Surgery. 1987; 30(2):79-80. http://europepmc.org/abstract/MED/3828914

[13] Nguyen JT, Nguyen JL, Wheatley MJ, Nguyen TA. Muscle hernias of the leg: A case report and comprehensive review of the literature. Canadian Journal of Plastic Surgery. 2013; 21(4):243-7. [DOI:10.1177/229255031302100408]

[14] Schechter DC, Waddell WR, Coppinger WR. Muscle Hernia: Twenty personal observations. The American Surgeon. 1963; 29:483-90. https://www.semanticscholar.org/paper/ e7321d8cf1317738d38c4c75d1

[15] Schwartz-Fernandes FA, Lew A, Gonzalez MD. Repair of forearm muscle herniation using local fascial flap: A case report. Cureus. 2019; 11(6):3-6. [DOI:10.7759/cureus.4881] 